\title{
Risk allocation under liquidity constraints
}

Citation for published version (APA):

Csóka, P., \& Herings, P. J. J. (2013). Risk allocation under liquidity constraints. Maastricht University, Graduate School of Business and Economics. GSBE Research Memoranda No. 057 https://doi.org/10.26481/umagsb.2013057

Document status and date:

Published: 01/01/2013

DOI:

10.26481/umagsb.2013057

Document Version:

Publisher's PDF, also known as Version of record

\section{Please check the document version of this publication:}

- A submitted manuscript is the version of the article upon submission and before peer-review. There can be important differences between the submitted version and the official published version of record.

People interested in the research are advised to contact the author for the final version of the publication, or visit the DOI to the publisher's website.

- The final author version and the galley proof are versions of the publication after peer review.

- The final published version features the final layout of the paper including the volume, issue and page numbers.

Link to publication

\footnotetext{
General rights rights.

- You may freely distribute the URL identifying the publication in the public portal. please follow below link for the End User Agreement:

www.umlib.nl/taverne-license

Take down policy

If you believe that this document breaches copyright please contact us at:

repository@maastrichtuniversity.nl

providing details and we will investigate your claim.
}

Copyright and moral rights for the publications made accessible in the public portal are retained by the authors and/or other copyright owners and it is a condition of accessing publications that users recognise and abide by the legal requirements associated with these

- Users may download and print one copy of any publication from the public portal for the purpose of private study or research.

- You may not further distribute the material or use it for any profit-making activity or commercial gain

If the publication is distributed under the terms of Article $25 \mathrm{fa}$ of the Dutch Copyright Act, indicated by the "Taverne" license above, 


\section{Maastricht University}

Péter Csóka,

P. Jean-J acques Herings

Risk allocation under liquidity constraints

$\mathrm{RM} / 13 / 057$

\section{GSBE}

Maastricht University School of Business and Economics

Graduate School of Business and Economics

P.O Box 616

NL- 6200 MD Maastricht

The Netherlands 


\title{
Risk Allocation under Liquidity Constraints*
}

\author{
Péter Csóka ${ }^{\dagger} \quad$ P. Jean-Jacques Herings ${ }^{\ddagger}$
}

September 9, 2013

\begin{abstract}
Risk allocation games are cooperative games that are used to attribute the risk of a financial entity to its divisions. In this paper, we extend the literature on risk allocation games by incorporating liquidity considerations. A liquidity policy specifies state-dependent liquidity requirements that a portfolio should obey. To comply with the liquidity policy, a financial entity may have to liquidate part of its assets, which is costly.

The definition of a risk allocation game under liquidity constraints is not straightforward, since the presence of a liquidity policy leads to externalities. We argue that the standard worst case approach should not be used here and present an alternative definition. We show that the resulting class of transferable utility games coincides with the class of totally balanced games. It follows from our results that also when taking liquidity considerations into account there is always a stable way to allocate risk.
\end{abstract}

Keywords: Market Microstructure, Coherent Measures of Risk, Market Liquidity, Portfolio Performance Evaluation, Risk Capital Allocation, Totally Balanced Games

\section{JEL Classification: C71, G10}

*We would like to thank Gábor Czigány and participants of SING8, the Annual Financial Market Liquidity Conference, the 6th Financial Risks International Forum and SAET2013 for helpful comments.

†Department of Finance, Corvinus University of Budapest and "Momentum" Game Theory Research Group, Centre for Economic and Regional Studies, Hungarian Academy of Sciences. E-mail: peter.csoka@uni-corvinus.hu. Péter Csóka thanks funding from HAS (LP-004/2010) and OTKA (PD 105859).

${ }^{\ddagger}$ Department of Economics, Maastricht university, P.O. Box 616, 6200 MD, Maastricht, The Netherlands. E-mail: P.Herings@maastrichtuniversity.nl. 


\section{Introduction}

If a financial enterprise (bank, insurance company, investment fund, etc.) consists of several divisions (individuals, products, subportfolios, risk factors, etc.), not only is it important to measure the risk of the entire financial enterprise properly, but also to attribute this risk to the divisions using a proper risk allocation method.

To measure risk appropriately, we apply coherent measures of risk (Artzner, Delbaen, Eber, and Heath, 1999), which are defined by four axioms: monotonicity, subadditivity, positive homogeneity, and translation invariance. Csóka, Herings, and Kóczy (2007) show that these axioms are supported by a natural general equilibrium approach to measure risk. Of these axioms, subadditivity is the most essential one, and captures the notion that the risk of the financial enterprise is at most as large as the sum of the risks of the divisions.

Risk or capital allocation has many applications: the division of capital reserves among business units by financial institutions; strategic decision making regarding new business lines; product pricing; performance measurement; the formation of risk limits; see Denault (2001), Kalkbrener (2005), Buch and Dorfleitner (2008), Homburg and Scherpereel (2008), Kim and Hardy (2009), and Csóka, Herings, and Kóczy (2009).

The various applications have the following question in common: how to allocate the risk of the financial enterprise over its constituents? A natural approach to answer this question comes from cooperative game theory. First one defines a risk allocation game, the cooperative game where a player is a portfolio and the payoffs of a coalition are negatively related to the risk of the coalition's portfolio. The risk of a portfolio is measured by a coherent measure of risk. Next, one can use one of the point-valued solution concepts in cooperative game theory like the Shapley value (Shapley, 1953) or the nucleolus (Schmeidler, 1969) to attribute risk to the players, or one of the set-valued solution concepts like the core (Gillies, 1959) to determine stable allocations of risk.

Liquidity is a major concern in financial markets. The usual mark-to-market valuation of assets does not take the assets' liquidity into account. Acerbi and Scandolo (2008) extend the axioms of coherent risk measures to incorporate two types of liquidity considerations. First, requirements on the composition of the portfolio, captured by the so-called liquidity policy and, second, the liquidity of asset markets as expressed by marginal demand curves, corresponding to the order books of the assets at a given future point in time.

In this paper we extend the notion of risk allocation games to include liquidity considerations. In doing so, the financial enterprise faces an externality problem, as any division of the enterprise can liquidate some of its assets in order to satisfy the liquidity policy, and the more assets one division liquidates, the less assets have to be liquidated by other divisions. The standard approaches to define a game in characteristic function form in the

presence of externalities are $\alpha$-effectiveness and $\beta$-effectiveness as suggested by Aumann 
(1961), thereby generalizing the two-player case treated by von Neumann and Morgenstern (1944) to the case with an arbitrary number of players.

The notion of $\alpha$-effectiveness defines the payoff of a coalition as the payoff it can achieve irrespective of the actions taken by its complement. In a sense, the coalition acts first in anticipation of the worst actions its complement can take. The notion of $\beta$-effectiveness is less stringent and defines the payoff of a coalition as what it can achieve for sure given the worst actions of its complement, so now the complementary coalition acts first. We will argue that as soon as the liquidity considerations are non-trivial, the two standard approaches should not be applied. We also argue that the approach where the portfolio of the coalition's complement is ignored, or equivalently, put equal to zero, is not satisfactory.

Rather than taking a worst-case approach for the behavior of the complementary coalition, we will fix the portfolio holdings of the players outside the coalition to their initial values, thereby placing the burden of satisfying the liquidity policy entirely on the coalition itself, but not putting an extra burden because of adversary behavior of the coalition's complement. The resulting cooperative games with transferable utility are called risk allocation games with liquidity constraints. Our main theorem claims that the class of risk allocation games with liquidity constraints are totally balanced, thereby generalizing the result for risk allocation games without liquidity constraints in Csóka, Herings, and Kóczy (2009). A direct consequence of this result is that risk allocation games with liquidity constraints have a non-empty core. Thus it follows that even when taking liquidity considerations into account, there is always a stable way to allocate risk, meaning that no coalition of players would object to it.

It has been shown in Csóka, Herings, and Kóczy (2009) that any totally balanced game is generated by some risk allocation game without liquidity constraints. Since a risk allocation game without liquidity considerations results as a special case when the liquidity policy is trivial and assets are perfectly liquid, it holds that any totally balanced game is generated by some risk allocation game with liquidity constraints. We therefore obtain an equivalence between the class of risk allocation games with liquidity constraints and the class of totally balanced games.

The structure of the paper is as follows. In Section 2 we set up risk environments with liquidity considerations and in Section 3 we define risk allocation games with liquidity constraints. Section 4 contains our main theorem and Section 5 concludes.

\section{Risk Environments with Liquidity Considerations}

Acerbi and Scandolo (2008) study coherent measures of risk in a framework where portfolios are subject to liquidity considerations. Csóka, Herings, and Kóczy (2009) study risk 
allocation games that are generated by coherent measures of risk in a set-up where liquidity considerations are absent. In this section we extend the analysis of Csóka, Herings, and Kóczy (2009) and we define risk environments that take liquidity considerations into account. We denote such risk environments by $(N, J, S, \pi, \theta, m, L, \rho)$.

The group of players in a risk environment is denoted by $N$, it consists of the $n$ divisions of a financial enterprise, and it is referred to as the firm. Each division holds cash as well as assets belonging to a set $J$. The initial portfolio $\theta^{i}=\left(\theta_{0}^{i}, \theta_{J}^{i}\right) \in \mathbb{R} \times \mathbb{R}^{J}$ of division $i \in N$ shows the amounts of cash and assets held initially by division $i$. The initial porfolio of the firm is given by the aggregate portfolio $\theta(N)=\sum_{i \in N} \theta^{i}$. We denote the space of portfolios by $P=\mathbb{R} \times \mathbb{R}^{J}$. Cash has a number of special properties which we explain in the sequel. The addition of an amount of cash $a \in \mathbb{R}$ to a portfolio $p \in P$ is denoted by $p \oplus a$ and results in the portfolio $q \in P$ defined by $q_{0}=p_{0}+a$ and $q_{j}=p_{j}, j \in J$. The tuple of the initial portfolios of the various divisions is denoted by $\theta=\left(\theta^{i}\right)_{i \in N}$.

The future value of the initial portfolio is subject to uncertainty. One out of a set $S$ of possible states of nature materializes in the future, where state of nature $s \in S$ occurs with probability $\pi_{s}>0$. Clearly, it holds that $\sum_{s \in S} \pi_{s}=1$. The value of the initial portfolio in state $s$ depends on the order books for the various assets and the liquidity policy of the firm, both of which are allowed to be state dependent. We follow Çetin, Jarrow and Protter (2004) and Jarrow and Protter (2005) in modeling the order book for asset $j$ in state $s$ by a marginal demand curve $m_{j}^{s}$. A function is càdlàg if it is right continuous with left limits and làdcàg if it is left continuous with right limits.

Definition 2.1. The marginal demand curve (MDC) for asset $j \in J$ in state $s \in S$ is given by the map $m_{j}^{s}: \mathbb{R} \backslash\{0\} \mapsto \mathbb{R}$ satisfying

(i) $m_{j}^{s}(x) \geq m_{j}^{s}\left(x^{\prime}\right)$ if $x<x^{\prime}$;

(ii) $m_{j}^{s}$ is càdlàg at $x<0$ and làdcàg at $x>0$.

The amount $m_{j}^{s}(x)$ for $x>0$ expresses the marginal bids that have been made to buy an amount $x$ of asset $j$. Similarly, $m_{j}^{s}(x)$ for $x<0$ represents the marginal asks that have been made for an amount $x$ of asset $j$ to be sold. We call $m^{s}\left(0_{j}^{+}\right)$the best bid and $m^{s}\left(0_{j}^{-}\right)$ the best ask price of asset $j$. Note that the MDC is not defined at zero.

So far the issue of liquidity has not been considered at all in risk allocation games. Implicitly, it has been assumed that the MDCs are all flat, corresponding to perfectly liquid asset markets.

Definition 2.2. Asset $j \in J$ is perfectly liquid if for every $s \in S$, there is $c \in \mathbb{R}$ such that for all $x \in \mathbb{R} \backslash\{0\}, m_{j}^{s}(x)=c$.

Since the constant $c$ is allowed to depend on $s$, the price against which the asset can be bought or sold is allowed to be stochastic, even when an asset market is perfectly liquid. 
The MDC can be used to calculate the liquidation value of a portfolio.

Definition 2.3. The liquidation mark-to-market value of a portfolio $p \in P$ in state $s \in S$ is defined by

$$
\ell^{s}(p)=p_{0}+\sum_{j \in J} \int_{0}^{p_{j}} m_{j}^{s}(x) d x .
$$

The liquidation mark-to-market value of a portfolio equals the portfolio's amount of cash plus the proceeds of selling all long positions minus the payments needed to close short positions. Acerbi and Scandolo (2008) prove the following result.

Proposition 2.4. For every $s \in S$, the function $\ell^{s}: P \mapsto \mathbb{R}$ is continuous and concave.

The set of portfolios attainable from some given portfolio $p \in P$ in state $s \in S$ is given by

$$
A^{s}(p)=\left\{q \in P \mid q_{0}=\ell^{s}\left(p_{0}, p_{J}-q_{J}\right)\right\} .
$$

The portfolio $q \in A^{s}(p)$ is obtained by liquidating the amounts $p_{J}-q_{J}$ of assets in $J$ and adding the proceeds to $p_{0}$.

Proposition 2.5. For every $s \in S$, for every $p \in P$, the set $A^{s}(p)$ is closed.

Proof. Let $\left(q^{r}\right)_{r \in \mathbb{N}}$ be a sequence in $A^{s}(p)$ with limit $\bar{q} \in P$. We have to show that $\bar{q} \in A^{s}(p)$. For every $r \in \mathbb{N}$, it holds that $q_{0}^{r}=\ell^{s}\left(p_{0}, p_{J}-q_{J}^{r}\right)$. By Proposition 2.4 it holds that $\ell^{s}$ is continuous, so

$$
\bar{q}_{0}=\ell^{s}\left(p_{0}, p_{J}-\bar{q}_{J}\right) .
$$

It now follows from the definition that $\bar{q} \in A^{s}(p)$.

The liquidity policy (Acerbi and Scandolo, 2008) incorporates the requirements imposed by a regulator or the contractual obligations that have to be met, and specifies that the portfolio of the firm should belong to the set $L^{s} \subset P$ in state of nature $s \in S$. We denote $L=\left(L^{s}\right)_{s \in S}$. The state dependence of the liquidity policy enables us to model regulatory risk as well as short sale constraints which depend on market conditions as expressed in the prevailing MDC.

The literature on risk allocation games has so far ignored liquidity policies.

Definition 2.6. The liquidity policy is trivial if for every $s \in S$ it holds that $L^{s}=P$. Throughout the paper we make the following assumption on $L$. 
Assumption 2.7. For every $s \in S$, it holds that

(i) $L^{s}$ is closed and convex;

(ii) for every $p \in L^{s}$, for every $a>0, p \oplus a \in L^{s}$;

(iii) $A^{s}(\theta(N)) \cap L^{s} \neq \emptyset$.

The first two items in Assumption 2.7 are inherited from Acerbi and Scandolo (2008). Closedness as required in Assumption 2.7.(i) is a standard technical assumption. Convexity means that if two portfolios are acceptable, then so is their weighted average. Assumption 2.7.(ii) implies that it is always acceptable to have more cash. Assumption 2.7.(iii) guarantees that in every state there is a feasible choice to meet the requirements of the liquidity policy. Assumption 2.7 is satisfied if the liquidity policy is trivial.

In case all assets are perfectly liquid, the liquidity policy is irrelevant. This is the case which has been studied in the existing literature on risk allocation games. Even if the liquidity policy is trivial, the liquidity of assets can matter if there is a bid-ask spread.

For a portfolio $p \in P$, we denote the assets hold long by $J^{+}(p)=\left\{j \in J \mid p_{j}>0\right\}$ and the assets hold short by $J^{-}(p)=\left\{j \in J \mid p_{j}<0\right\}$.

Definition 2.8. The uppermost mark-to-market value of a portfolio $p \in P$ in state $s \in S$ is defined by

$$
u^{s}(p)=p_{0}+\sum_{j \in J^{+}(p)} m_{j}^{s}\left(0^{+}\right) p_{j}+\sum_{j \in J^{-}(p)} m_{j}^{s}\left(0^{-}\right) p_{j} .
$$

The uppermost mark-to-market value of a portfolio can be interpreted as the value of a portfolio in the long run. Long positions are valued using the best bid prices and short positions using the best ask prices. In case all asset markets are perfectly liquid, the uppermost mark-to-market value of a portfolio is equal to its liquidation mark-to-market value. Acerbi and Scandolo (2008) prove the following result.

Proposition 2.9. For every $s \in S$, the function $u^{s}: P \mapsto \mathbb{R}$ is continuous, concave, and positive homogeneous of degree one.

Two portfolios $p, q \in P$ are said to be concordant if $p_{j} q_{j} \geq 0$ for all $j \in J$. Two portfolios are concordant if there is no asset which is held long in one portfolio and short in the other. It is easily verified that $u^{s}$ is additive for concordant portfolios. The concavity and positive homogeneity of $u^{s}$ imply that $u^{s}$ is superadditive on $P$, i.e. $u^{s}(p)+u^{s}(q) \leq u^{s}(p+q)$ for all $p, q \in P$.

Corollary 2.10. For every $s \in S$, the function $u^{s}$ is superadditive on $P$ and additive for concordant portfolios. 
Given some state $s \in S$, the firm might have to liquidate part of its assets to obtain a portfolio in $L^{s}$. The initial portfolio's value in state $s$ is given by the highest attainable uppermost mark-to-market value satisfying the liquidity policy. These considerations lead us to the following definition.

Definition 2.11. The realization vector $X(N) \in \mathbb{R}^{S}$ for the firm is equal to

$$
X^{s}(N)=\sup \left\{u^{s}(q) \mid q \in A^{s}(\theta(N)) \cap L^{s}\right\}, \quad s \in S .
$$

Since $A^{s}(\theta(N)) \cap L^{s} \neq \emptyset$ by Assumption 2.7, it follows that $X^{s}(N)$ is not equal to $-\infty$. We will show in Proposition 3.5 that $X^{s}(N)$ is bounded from above by $u^{s}(\theta(N))$, so $X^{s}(N)$ is finite.

The following example shows why in Equation (3) we need a supremum rather than a maximum.

Example 2.12. Consider the case where $\theta(N)=\left(\theta_{0}(N), \theta_{J}(N)\right) \in \mathbb{R}^{3}$, so we have two assets, called 1 and 2, and cash. There is no uncertainty, so the cardinality of $S$ is one. The liquidity policy $L$ specifies that the asset portfolio $q_{J}$ should satisfy $q_{J} \geq(-1,-1)$ and $\left(q_{1}+1\right)\left(q_{2}+1\right) \geq 1$. Moreover, for simplicity assume there are no constraints on cash holdings. We assume the initial asset portfolio to be $\theta(N)=(0,-1,-1)$ and we assume asset 2 to be perfectly liquid.

Trading in asset 1 involves liquidity costs. For instance, consider the case where there is a simple bid-ask spread, so for some $\gamma>0, m_{1}(x)=1+\gamma$ if $x<0$ and $m_{1}(x)=1$ if $x>0$. Since we have to go to a portfolio of assets where holdings of both assets strictly exceed -1 , we would like to buy $\varepsilon>0$ of asset 1 , buy $1 / \varepsilon$ of asset 2 , and go short in cash, resulting in a portfolio $q(\varepsilon)=\left(-(1+\gamma) \varepsilon-m_{2}\left(0^{-}\right) / \varepsilon,-1+\varepsilon,-1+1 / \varepsilon\right)$. It is straightforward to verify that $u(q(\varepsilon))=u(\theta(N))-\gamma \varepsilon$. The uppermost mark-to-market value would be maximized, and liquidity costs would be minimized, by taking the smallest positive $\varepsilon$, something which clearly does not exist, so we need a supremum rather than a maximum in Equation (3).

We will show in Proposition 3.6 that optimal portfolios exist under the mild and reasonable additional assumption that going infinitely short or long involves liquidity costs. Under such an additional assumption, one can use a maximum in Equation (3).

Artzner, Delbaen, Eber, and Heath (1999) have introduced coherent measures of risk. A measure of risk is a function $\rho: \mathbb{R}^{S} \rightarrow \mathbb{R}$ measuring the risk of a realization vector from the perspective of the present. It corresponds to the minimal amount of cash the regulated agent has to add to his portfolio, and to invest in a reference instrument today, such that the risk involved in the portfolio is acceptable to the regulator. We assume that the reference instrument has payoff 1 in each state of nature at $t=1$, thus its realization vector is $1^{S}=(1, \ldots, 1)$. It is most natural to think of it as a zero coupon bond. The price of the reference instrument can be thought of as the discount factor and is denoted by $\delta$. 
Definition 2.13. A function $\rho: \mathbb{R}^{S} \rightarrow \mathbb{R}$ is a coherent measure of risk if it satisfies the following axioms.

1. Monotonicity: for all $X, Y \in \mathbb{R}^{S}$ such that $Y \geq X$, we have $\rho(Y) \leq \rho(X)$.

2. Subadditivity: for all $X, Y \in \mathbb{R}^{S}$, we have $\rho(X+Y) \leq \rho(X)+\rho(Y)$.

3. Positive homogeneity: for all $X \in \mathbb{R}^{S}$ and $h \in \mathbb{R}_{+}$, we have $\rho(h X)=h \rho(X)$.

4. Translation invariance: for all $X \in \mathbb{R}^{S}$ and $a \in \mathbb{R}$, we have $\rho\left(X+a 1^{S}\right)=\rho(X)-\delta a$.

This completes the definition of a risk environment with liquidity considerations $(N, J, S, \pi, \theta, m, L, \rho)$.

\section{Risk Allocation Games with Liquidity Constraints}

A cooperative game with transferable utility consists of a set of players $N$ and a value function $v: 2^{N} \rightarrow \mathbb{R}$, which assigns to every coalition $C \in 2^{N}$ of players a worth $v(C) \in \mathbb{R}$. By assumption it holds that $v(\emptyset)=0$. An allocation is a vector $y \in \mathbb{R}^{n}$, where $y_{i}$ is the payoff of player $i \in N$. An allocation $y$ yields payoff $y(C)=\sum_{i \in C} y_{i}$ to the members of coalition $C$. An allocation $y \in \mathbb{R}^{n}$ is called efficient if $y(N)=v(N)$, individually rational if $y_{i} \geq v(\{i\})$ for all $i \in N$, and coalitionally rational if $y(C) \geq v(C)$ for all $C \in 2^{N}$. The core is the set of efficient and coalitionally rational allocations.

Denault (2001) introduces risk capital allocation problems. The question is how the risk of the firm as measured by a coherent measure of risk has to be attributed to its divisions. The risk allocated to a coalition of divisions $C \in 2^{N}$ should be stable, meaning that it does not exceed the risk of the aggregate portfolio of coalition $C$.

Denault (2001) abstracts both from MDCs and liquidity policies, and thereby implicitly assumes that all assets are perfectly liquid and the liquidity policy is trivial. Under perfect liquidity of all assets and a trivial liquidity policy, we can define the realization vector of division $i \in N$ by $X^{s}(\{i\})=u^{s}\left(\theta^{i}\right), s \in S$. A coalition of divisions $C \in 2^{N}$ has

the realization vector $X(C)=\sum_{i \in C} X(\{i\})$. Finally, the worth of coalition $C$ is defined by $v(C)=-\rho(X(C))$. In this way we have obtained a cooperative game with transferable utility $(N, v)$. Standard solution concepts from cooperative game theory can now be applied to $(N, v)$ to solve the risk allocation problem. A stable risk allocation corresponds to a core allocation.

When we incorporate liquidity constraints, we face an externality problem, as any division of the firm can liquidate some of its assets in order to satisfy the liquidity policy, and the more assets one division liquidates, the less assets have to be liquidated by other 
divisions. The standard approaches to define a game in characteristic function form in the presence of externalities are $\alpha$-effectiveness and $\beta$-effectiveness as suggested by Aumann (1961), thereby generalizing the two-player case treated by von Neumann and Morgenstern (1944) to the case with an arbitrary number of players. The notion of $\alpha$-effectiveness defines the payoff of a coalition as the payoff it can achieve irrespective of the actions taken by its complement. The next example illustrates why $\alpha$-effectiveness is not useful to study risk allocation in the presence of liquidity constraints.

Example 3.1. Consider the case where we have one asset, $J=\{1\}$, and no uncertainty. The liquidity policy $L$ specifies that the firm's portfolio $q$ should satisfy $q_{1} \geq-1$. The firm has two divisions, $N=\{1,2\}$, with identical initial portfolios given by $\theta^{1}=(1,-1)$ and $\theta^{2}=(1,-1)$. Consider the case where there is a simple bid-ask spread, so for some $\gamma>0$, we have $m_{1}(x)=1+\gamma$ if $x<0$ and $m_{1}(x)=1$ if $x>0$. Under $\alpha$-effectiveness, division 1's realization is equal to

$$
X(\{1\})=\sup \left\{\underline{u} \in \mathbb{R} \mid \exists q^{1} \in A\left(\theta^{1}\right), \forall q^{2} \in A\left(\theta^{2}\right), q^{1}+q^{2} \in L \text { and } u\left(q^{1}\right) \geq \underline{u}\right\} .
$$

For $i=1,2$, we have that $q^{i} \in A\left(\theta^{i}\right)$ if and only if

$$
\left[q_{1}^{i} \geq-1 \text { and } q_{0}^{i}=1-\left(q_{1}^{i}+1\right)(1+\gamma)\right] \text { or }\left[q_{1}^{i} \leq-1 \text { and } q_{0}^{i}=-q_{1}^{i}\right]
$$

Since for any choice of $q^{1} \in A\left(\theta^{1}\right)$ there is $q^{2} \in A\left(\theta^{2}\right)$ such that $q^{1}+q^{2} \notin L$, in fact any $q^{2}$ such that $q_{1}^{2}<-q_{1}^{1}-1$ would do, we find that $X(\{1\})=-\infty$. Notice that the same conclusion would follow even in the absence of a bid-ask spread.

The notion of $\beta$-effectiveness is less stringent and defines the payoff of a coalition as what it can achieve for sure given the worst actions of its complement, so now the complementary coalition acts first. We continue Example 3.1 by demonstrating that also $\beta$-effectiveness leads to undesirable consequences.

Example 3.2. Consider the primitives of Example 3.1. Under $\beta$-effectiveness, division 1's realization is equal to

$$
X(\{1\})=\sup \left\{\underline{u} \in \mathbb{R} \mid \forall q^{2} \in A\left(\theta^{2}\right), \exists q^{1} \in A\left(\theta^{1}\right), q^{1}+q^{2} \in L \text { and } u\left(q^{1}\right) \geq \underline{u}\right\} .
$$

Consider some $q^{2} \in A\left(\theta^{2}\right)$ with $q_{1}^{2} \leq-1$. To satisfy $q^{1}+q^{2} \in L$, it should hold that $q_{1}^{1} \geq-q_{1}^{2}-1$ and therefore that $q_{1}^{1} \geq 0$. It can easily be computed that

$$
u\left(q^{1}\right)=-\gamma-\gamma q_{1}^{1} \leq-\gamma+\gamma\left(q_{1}^{2}+1\right)=\gamma q_{1}^{2} .
$$

Since $q_{1}^{2}$ can be chosen arbitrarily negative, we find that $X(\{1\})=-\infty$. 
Both $\alpha$-effectiveness and $\beta$-effectiveness lead to undesirable properties of the value function, even in the simplest of examples. Both approaches share the feature that the complement of a coalition $C$ is supposed to take the worst possible action. Rather than making such an extreme assumption, we will instead assume that the complement of coalition $C$ remains inactive, so the burden of satisfying the liquidity policy will be put entirely on coalition $C$. The portfolios which are attainable for coalition $C$ in state $s \in S$ are given by $A^{s}(\theta(C))$, where $\theta(C)=\sum_{i \in C} \theta^{i}$. Inactivity of the complementary coalition means that those divisions stick to their initial portfolio, which equals $\theta(N \backslash C)$ in the aggregate.

Definition 3.3. Given a risk environment with liquidity considerations $(N, J, S, \pi, \theta, m, L, \rho)$ and a coalition of divisions $C \in 2^{N}$, the realization vector $X(C)$ of coalition $C$ is defined by

$$
X^{s}(C)=\sup \left\{u^{s}(q) \mid q \in A^{s}(\theta(C)) \text { and } q+\theta(N \backslash C) \in L^{s}\right\}, \quad s \in S .
$$

When calculating $X^{s}(C)$, we take the portfolios of the divisions outside the coalition as fixed, and liquidate the portfolios of the divisions in $C$ in such a way that the resulting portfolio of the firm is attainable and satisfies the liquidity policy. Applying Definition 3.3 for the grand coalition $N$ results in

$$
X^{s}(N)=\sup \left\{u^{s}(q) \mid q \in A^{s}(\theta(N)) \cap L^{s}\right\},
$$

which is in accordance with Equation (3).

The next result is useful in simplifying our expression for the realization vector of a coalition.

Proposition 3.4. For every $s \in S$, for every $C \in 2^{N}$, it holds that $q \in A^{s}(\theta(C))$ if and only if $q+\theta(N \backslash C) \in A^{s}(\theta(N))$.

Proof. The result follows from the observation that

$$
q \in A^{s}(\theta(C))
$$

if and only if

$$
q_{0}=\ell^{s}\left(\theta_{0}(C), \theta_{J}(C)-q_{J}\right)=\theta_{0}(C)+\ell^{s}\left(0, \theta_{J}(C)-q_{J}\right)
$$

if and only if

$$
\begin{aligned}
q_{0}+\theta_{0}(N \backslash C) & =\theta_{0}(C)+\ell^{s}\left(0, \theta_{J}(C)-q_{J}\right)+\theta_{0}(N \backslash C) \\
& =\ell^{s}\left(\theta_{0}(N), \theta_{J}(N)-q_{J}-\theta_{J}(N \backslash C)\right)
\end{aligned}
$$

if and only if

$$
q+\theta(N \backslash C) \in A^{s}(\theta(N)) .
$$


Proposition 3.4 shows that we can compute the realization vector of coalition $C$ as

$$
X^{s}(C)=\sup \left\{u^{s}(q) \mid q+\theta(N \backslash C) \in A^{s}(\theta(N)) \cap L^{s}\right\}, \quad s \in S .
$$

The next result provides bounds on the value of $X^{s}(C)$, implying that this value is not equal to $-\infty$ or $+\infty$.

Proposition 3.5. For every $s \in S$, for every $q \in A^{s}(\theta(N)) \cap L^{s}$, it holds that

$$
u^{s}(q-\theta(N \backslash C)) \leq X^{s}(C) \leq u^{s}(\theta(C)), \quad C \in 2^{N} .
$$

Proof. Consider some $q \in A^{s}(\theta(N)) \cap L^{s}$, where the latter set is non-empty by Assumption 2.7. Using (4), it follows that $u^{s}(q-\theta(N \backslash C)) \leq X^{s}(C)$.

Next, consider some $q \in A^{s}(\theta(C))$. It holds that

$$
q_{0}=\ell^{s}\left(\theta_{0}(C), \theta_{J}(C)-q_{J}\right)=\theta_{0}(C)+\sum_{j \in J} \int_{0}^{\theta_{j}(C)-q_{j}} m_{j}^{s}(x) d x
$$

It follows that

$$
\begin{aligned}
u^{s}(q)= & \theta_{0}(C)+\sum_{j \in J} \int_{0}^{\theta_{j}(C)-q_{j}} m_{j}^{s}(x) d x+\sum_{j \in J^{+}(q)} m_{j}^{s}\left(0^{+}\right) q_{j}+\sum_{j \in J^{-}(q)} m_{j}^{s}\left(0^{-}\right) q_{j} \\
= & \theta_{0}(C)+\sum_{j \in J^{+}(q)}\left(\int_{0}^{\theta_{j}(C)-q_{j}} m_{j}^{s}(x) d x+m_{j}^{s}\left(0^{+}\right) q_{j}\right) \\
& +\sum_{j \in J^{-}(q)}\left(\int_{0}^{\theta_{j}(C)-q_{j}} m_{j}^{s}(x) d x+m_{j}^{s}\left(0^{-}\right) q_{j}\right) .
\end{aligned}
$$

Notice that irrespective of the sign of $\theta_{j}(C)-q_{j}$ it holds that

$$
\int_{0}^{\theta_{j}(C)-q_{j}} m_{j}^{s}(x) d x \leq m_{j}^{s}\left(0^{-}\right)\left(\theta_{j}(C)-q_{j}\right) \text { and } \int_{0}^{\theta_{j}(C)-q_{j}} m_{j}^{s}(x) d x \leq m_{j}^{s}\left(0^{+}\right)\left(\theta_{j}(C)-q_{j}\right) .
$$

Consider some $j \in J^{+}(q)$. If $\theta_{j}(C)>0$, then

$$
\int_{0}^{\theta_{j}(C)-q_{j}} m_{j}^{s}(x) d x+m_{j}^{s}\left(0^{+}\right) q_{j} \leq m_{j}^{s}\left(0^{+}\right)\left(\theta_{j}(C)-q_{j}\right)+m_{j}^{s}\left(0^{+}\right) q_{j}=m_{j}^{s}\left(0^{+}\right)\left(\theta_{j}(C)\right) .
$$

If $\theta_{j}(C)<0$, then

$$
\int_{0}^{\theta_{j}(C)-q_{j}} m_{j}^{s}(x) d x+m_{j}^{s}\left(0^{+}\right) q_{j} \leq m_{j}^{s}\left(0^{-}\right)\left(\theta_{j}(C)-q_{j}\right)+m_{j}^{s}\left(0^{-}\right) q_{j}=m_{j}^{s}\left(0^{-}\right)\left(\theta_{j}(C)\right) .
$$

Consider some $j \in J^{-}(q)$. If $\theta_{j}(C)>0$, then

$$
\int_{0}^{\theta_{j}(C)-q_{j}} m_{j}^{s}(x) d x+m_{j}^{s}\left(0^{-}\right) q_{j} \leq m_{j}^{s}\left(0^{+}\right)\left(\theta_{j}(C)-q_{j}\right)+m_{j}^{s}\left(0^{+}\right) q_{j}=m_{j}^{s}\left(0^{+}\right)\left(\theta_{j}(C)\right) .
$$


If $\theta_{j}(C)<0$, then

$$
\int_{0}^{\theta_{j}(C)-q_{j}} m_{j}^{s}(x) d x+m_{j}^{s}\left(0^{-}\right) q_{j} \leq m_{j}^{s}\left(0^{-}\right)\left(\theta_{j}(C)-q_{j}\right)+m_{j}^{s}\left(0^{-}\right) q_{j}=m_{j}^{s}\left(0^{-}\right)\left(\theta_{j}(C)\right) .
$$

We therefore find that

$$
u^{s}(q) \leq \theta_{0}(C)+\sum_{j \in J^{+}(\theta(C))} m_{j}^{s}\left(0^{+}\right) \theta_{j}(C)+\sum_{j \in J^{-}(\theta(C))} m_{j}^{s}\left(0^{-}\right) \theta_{j}(C)=u^{s}(\theta(C)),
$$

which completes the proof.

If the liquidity policy is trivial, then it holds that $\theta(N) \in A^{s}(\theta(N)) \cap L^{s}$, so Proposition 3.5 gives $X^{s}(C)=u^{s}(\theta(C))$. If, moreover, there are no bid-ask spreads, it holds that $X^{s}(C)=\sum_{i \in C} X^{s}(\{i\})$, the case which has been studied in the literature so far. In case the liquidity policy is trivial, but assets are not perfectly liquid and there is a bid-ask spread, it is still the case that the realization vector of division $i \in N$ is given by $X^{s}(\{i\})=u^{s}\left(\theta^{i}\right)$, $s \in S$, but it is no longer necessarily the case that the realization vector of a coalition of divisions $C \in 2^{N}$ is given by $X(C)=\sum_{i \in C} X(\{i\})$ as now coalitions can save on costs related to bid-ask spreads by combining their portfolios.

We show next that the supremum in Equation (4) can be replaced by a maximum under the mild condition that going infinitely short or long in an asset involves liquidity costs.

Proposition 3.6. Assume that for every $s \in S$, for every $j \in J$, there is $x^{-}$such that $m_{j}^{s}\left(x^{-}\right)>m_{j}^{s}\left(0^{+}\right)$and there is $x^{+}$such that $m_{j}^{s}\left(x^{+}\right)<m_{j}^{s}\left(0^{-}\right)$. Then for every $C \in 2^{N}$ it holds that

$$
X^{s}(C)=\max \left\{u^{s}(q) \mid q+\theta(N \backslash C) \in A^{s}(\theta(N)) \cap L^{s}\right\}, \quad s \in S .
$$

Proof. Consider some $s \in S$ and let $\left(q^{r}\right)_{r \in \mathbb{N}}$ be a sequence such that $\left(q^{r}\right)_{r \in \mathbb{N}}+\theta(N \backslash C) \in$ $A^{s}(\theta(N)) \cap L^{s}$ and $u^{s}\left(q^{r}\right)$ converges to $X^{s}(C)$.

Suppose there is an asset $j^{\prime} \in J$ such that for some appropriately chosen subsequence, $\lim _{r \rightarrow \infty}\left(q_{j^{\prime}}^{r}\right)_{r \in \mathbb{N}}=-\infty$. We will derive a contradiction. By repeating the steps in the proof of Proposition 3.5, we derive that

$$
\begin{aligned}
u^{s}\left(q^{r}\right) \leq & \theta_{0}(C)+\sum_{j \in J^{+}(\theta(C))} m_{j}^{s}\left(0^{+}\right) \theta_{j}(C)+\sum_{j \in J^{-}(\theta(C)) \backslash\left\{j^{\prime}\right\}} m_{j}^{s}\left(0^{-}\right) \theta_{j}(C) \\
& +\int_{0}^{\theta_{j^{\prime}}(C)-q_{j^{\prime}}^{r}} m_{j^{\prime}}^{s}(x) d x+m_{j^{\prime}}^{s}\left(0^{-}\right) q_{j^{\prime}}^{r} \\
\leq & \theta_{0}(C)+\sum_{j \in J^{+}(\theta(C))} m_{j}^{s}\left(0^{+}\right) \theta_{j}(C)+\sum_{j \in J^{-}(\theta(C)) \backslash\left\{j^{\prime}\right\}} m_{j}^{s}\left(0^{-}\right) \theta_{j}(C) \\
& +m_{j^{\prime}}^{s}\left(x^{+}\right) \theta_{j^{\prime}}(C)+\left(m_{j^{\prime}}^{s}\left(0^{-}\right)-m_{j^{\prime}}^{s}\left(x^{+}\right)\right) q_{j^{\prime}}^{r},
\end{aligned}
$$

where the right-hand side tends to $-\infty$ if $r \rightarrow \infty$ since $m_{j^{\prime}}^{s}\left(0^{-}\right)>m_{j^{\prime}}^{s}\left(x^{+}\right)$. This contradicts the conclusion of Proposition 3.5, which establishes that $X^{s}(C)$ is bounded from below. 
We have shown that for every $j \in J$ there is no subsequence of $\left(q^{r}\right)_{r \in \mathbb{N}}$ such that $\lim _{r \rightarrow \infty}\left(q_{j}^{r}\right)_{r \in \mathbb{N}}=-\infty$. By the straightforward analogous argument, we can show that for every $j \in J$ there is no subsequence of $\left(q^{r}\right)_{r \in \mathbb{N}}$ such that $\lim _{r \rightarrow \infty}\left(q_{j}^{r}\right)_{r \in \mathbb{N}}=\infty$. It follows that the sequence $\left(q_{J}^{r}\right)_{r \in \mathbb{N}}$ is bounded. By continuity of $\ell^{s}$ on $P$ as asserted in Proposition 2.4, it follows that the sequence $\left(q_{0}^{r}\right)_{r \in \mathbb{N}}$ is bounded. Without loss of generality, the sequence $\left(q^{r}\right)_{r \in \mathbb{N}}$ can be assumed to converge to some $\bar{q} \in P$. The set $A^{s}(\theta(N)) \cap L^{s}$ is closed as the intersection of two sets which are closed by Proposition 2.5 and Assumption 2.7. It follows that $\bar{q}+\theta(N \backslash C) \in A^{s}(\theta(N)) \cap L^{s}$. By Proposition 2.9 it holds that $u^{s}$ is continuous, so $X^{s}(C)=u^{s}(\bar{q})$.

Since the existing literature on risk allocation assumes perfect liquidity, and we would like to incorporate perfect liquidity as a special case, we refrain from assumptions additional to Assumption 2.7, and continue with the formulation of Equation (4) involving a supremum.

Definition 3.7. Given a risk environment with liquidity considerations $(N, J, S, \pi, \theta, m, L, \rho)$, the risk allocation game with liquidity constraints is the game $(N, v)$, where the value function $v: 2^{N} \rightarrow \mathbb{R}$ is defined by

$$
v(C)=-\rho(X(C)), \quad C \in 2^{N} .
$$

Let $\Gamma_{\mathrm{rl}}$ denote the family of risk allocation games with liquidity constraints with set of players $N$. In such a game, according to Equation (5), the larger the risk of any subset of portfolios, the lower its worth.

Example 3.8. Consider a firm with $n=2$ divisions, where each division has invested into one asset and cash, $J=\{1\}$. The portfolios of the divisions are $\theta^{1}=(20,-6)$ and $\theta^{2}=$ $(26,-7)$, so both divisions have short positions in the risky asset. We assume $S=\{1,2\}$ with both states having equal probability of occurrence.

In state 1 the MDC of the risky asset is given by

$$
m_{1}^{1}(x)= \begin{cases}5 & \text { if } x<0 \\ 4 & \text { if } x>0\end{cases}
$$

In state 2 the MDC of the risky asset is given by

$$
m_{1}^{2}(x)= \begin{cases}10 & \text { if } x<-2 \\ 9 & \text { if }-2 \leq x<-1 \\ 8 & \text { if }-1 \leq x<0 \\ 7 & \text { if } 0<x \leq 1 \\ 6 & \text { if } 1<x\end{cases}
$$


We consider the deterministic liquidity policy specified by $L^{1}=L^{2}=\left\{p \in P \mid p_{1} \geq\right.$ $-10\}$. In both states the liquidity policy of the firm does not allow to short the risky asset by more than 10 units. According to the initial porfolios, the two divisions together are $6+7=13$ units short in the risky asset. We set $\delta=1$ and take the maximum loss as the coherent measure of risk, so $\rho(X)=\max _{s \in S}-X^{s}$.

First, let us consider state 1 . This element of the realization vector of division 1 is calculated as follows. Looking at the MDC of the risky asset in state 1, we see that the firm can buy the risky asset for a price of 5 , and it can sell more risky assets for a price of 4 . Since currently the two divisions together are shorting 13 units from the risky asset and the liquidity policy allows to short at most 10 units, at least 3 units should be bought. If 3 units are bought for a price of $5 \times 3=15$, division 1 ends up with the portfolio $q^{1}(\{1\})=(5,-3)$ in state 1 by trading $t^{1}(\{1\})=(-15,3)$. The uppermost mark-to-market value of $q^{1}(\{1\})$ is $u^{1}\left(q^{1}(\{1\})\right)=5-3 \times 5=-10$. It is easy to check that we get the same value if division 1 buys anywhere between 3 to 6 units from the risky asset. Due to the bid-ask spread, buying back more than 6 units would result in a loss of 1 for each additional unit, since long positions would be valued at 4 per unit. It follows that the realization vector of division 1 in state 1 is $X^{1}(\{1\})=-10$. Similarly, the realization vector of division 2 in state 1 is $X^{1}(\{2\})=-9$. For the firm itself, we have $\theta^{1}+\theta^{2}=(46,-13)$, and an optimal portfolio $q^{1}(\{1,2\})$ satisfying the liquidity constraint is anywhere between $(31,-10)$ and $(-19,0)$, resulting in $X^{1}(\{1,2\})=-19$.

Next, let us analyze state 2. Again, division 1 should buy at least 3 units of the risky asset to satisfy the liquidity policy. Buying 3 units and trading $t^{2}(\{1\})=(-27,3)$ will result in $q^{2}(\{1\})=(-7,-3)$ and $u^{2}\left(q^{2}(\{1\})\right)=-7-3 \times 8=-31$. Buying more than 3 units would be costly. For instance, buying 4 units by trading $\bar{t}^{2}(\{1\})=(-37$, 4) would result in $\bar{q}^{2}(\{1\})=(-17,-2)$ and $u^{2}\left(\bar{q}^{2}(\{1\})\right)=-17-2 \times 8=-33$, hence division 1 will only buy 3 units. The remaining short position will be valued at the best ask price, and $X^{2}(\{2\})=-31$. Similarly, the realization vector of division 2 in state 2 is $X^{2}(\{2\})=-33$. For the firm itself, since $\theta^{1}+\theta^{2}=(46,-13)$, the optimal trade is $t^{2}(\{1,2\})=(-27,3)$, leading to $q^{2}(\{1,2\})=(19,-10)$ and $X^{2}(\{1,2\})=u^{2}\left(q^{2}(\{1,2\})\right)=19-10 \times 8=-61$. The calculations and the resulting cooperative game are summarized in Table 1.

Note that in state 1 the realization vector of coalition $\{1,2\}$ is additive over its members' realization vectors, which is due to the flat MDCs and the concordant portfolios. In state 2 coalition $\{1,2\}$ gains since it only has to buy back once 3 units of the risky asset at a higher price. Also note that if the original portfolio of the grand coalition satisfies the liquidity policy and the portfolios of the divisions are concordant, then we get additivity for the realization vector. On the other hand, if for the same asset with a positive bid-ask spread some divisions have had long positions and some other divisions have had short 


\begin{tabular}{c|rrr} 
state $/ X(C)$ & $X(\{1\})$ & $X(\{2\})$ & $X(\{1,2\})$ \\
\hline$s=1$ & -10 & -9 & -19 \\
$s=2$ & -31 & -33 & -61 \\
\hline$\rho(X(C))$ & 31 & 33 & 61 \\
$v(C)$ & -31 & -33 & -61
\end{tabular}

Table 1: A risk allocation game with liquidity constraints.

positions, then we would get superadditivity.

For two-player games, most single-valued solution concepts, and in particular the Shapley value and the nucleolus, would share the surplus of the grand coalition over the individual worths equally over the players. In this example, this would result in the allocation $(-29.5,-31.5)$. The firms should add 61 units of cash to its portfolio to make it acceptable to the regulator, and the risk allocation over the divisions corresponds to 29.5 units of cash for division 1 and 31.5 units of cash for division 2 .

Rather than assuming the worst-case for the behavior of the opponents, we have utilized the fact that in a risk environment with liquidity considerations, divisions have initial portfolios, which means that it is meaningful to speak about inaction of a division. We have calculated the realization vector of a coalition by assuming inactivity of the complementary coalition.

Another alternative which comes to mind when defining the realization vector of a coalition, is to assume that the complementary coalition is going to hold the 0 portfolio. Alternatively, one could think of this assumption as ignoring the complementary coalition. It is not hard to see that this is not an attractive alternative. Consider for instance the risk environment with liquidity considerations of Example 3.8. When the complementary coalition holds the 0 portfolio, it is easy to calculate that $v(\{1\})=-28, v(\{2\})=-30$, and $v(\{1,2\})=-61$. The resulting risk allocation game with liquidity constraints is not superadditive. The reason is that in this example, it is easier for a single division to satisfy the short-sales constraint of 10 units than it is for the entire firm. Failure of superadditivity is not natural for the problem under consideration, as the entire firm has more actions at its disposal to satisfy the liquidity policy than a single division.

\section{Total Balancedness}

For each $C \in 2^{N}$, let $a(C) \in \mathbb{R}^{n}$ be the membership vector, $a_{i}(C)=1$ for $i \in C$ and $a_{i}(C)=0$ otherwise. 
Definition 4.1. A balanced vector of weights is a vector $\left(\lambda^{C}\right)_{C \in 2^{N}} \in \mathbb{R}_{+}^{2^{N}}$ such that $\sum_{C \in 2^{N}} \lambda^{C} a(C)=a(N)$. A game $(N, v)$ is balanced if $\sum_{C \in 2^{N}} \lambda^{C} v(C) \leq v(N)$ for all balanced vectors of weights.

A well-known interpretation of balancedness is that the players can distribute one unit of working time to any coalition and if each coalition is active during a fraction $\lambda^{C}$ of a unit of time, then the players cannot generate more value than $v(N)$, the value of the grand coalition. Balancedness is a necessary and sufficient condition for non-emptiness of the core in a transferable utility game (Bondareva, 1963; Shapley, 1967). See Predtetchinski and Herings (2004) for an extension of the concept of balancedness to be necessary and sufficient for non-emptiness of the core in non-transferable utility games.

For a game $(N, v)$ and a coalition $C \in 2^{N}$, a subgame $\left(C, v^{C}\right)$ is obtained by restricting $v$ to subsets of $C$.

Definition 4.2. A game $(N, v)$ is totally balanced if for every $D \in 2^{N}$ its subgame $\left(D, v^{D}\right)$ is balanced, that is, if for all $D \in 2^{N}$ and for all vectors $\left(\lambda^{C}\right)_{C \in 2^{D}} \in \mathbb{R}_{+}^{2^{D}}$ satisfying $\sum_{C \in 2^{D}} \lambda^{C} a(C)=a(D)$, we have $\sum_{C \in 2^{D}} \lambda^{C} v(C) \leq v(D)$.

In a totally balanced game, every subgame has a non-empty core. Let $\Gamma_{\text {tb }}$ denote the family of totally balanced games with $n$ players.

The next proposition claims that any risk allocation game with liquidity constraints is totally balanced.

Proposition 4.3. All games $(N, v) \in \Gamma_{\mathrm{rl}}$ are totally balanced, $\Gamma_{\mathrm{rl}} \subset \Gamma_{\mathrm{tb}}$.

Proof. Consider a risk environment with liquidity considerations $(N, J, S, \pi, \theta, m, L, \rho)$ inducing the risk allocation game $(N, v)$. We show that for any non-empty $D \in 2^{N}$, the subgame $\left(D, v^{D}\right)$ is balanced. We define $\mathcal{D}=2^{D} \backslash\{\emptyset\}$. Take any $\left(\lambda^{C}\right)_{C \in \mathcal{D}} \in \mathbb{R}_{+}^{\mathcal{D}}$ such that $\sum_{C \in \mathcal{D}} \lambda^{C} a(C)=a(D)$.

Take $\varepsilon>0$. For $s \in S$ and $C \in \mathcal{D}$, let $q^{s}(C) \in P$ be such that $q^{s}(C)+\theta(N \backslash C) \in$ $A^{s}(\theta(N)) \cap L^{s}$ and

$$
u^{s}\left(q^{s}(C)\right) \geq X^{s}(C)-\varepsilon .
$$

The vector of actual trades is given by

$$
t^{s}(C)=q^{s}(C)-\theta(C) .
$$

First, we present eight lines containing equalities and inequalities proving the proposition, then we explain why each step is true. 
It holds that

$$
\begin{aligned}
\sum_{C \in \mathcal{D}} \lambda^{C} v^{D}(C) & =-\sum_{C \in \mathcal{D}} \lambda^{C} \rho(X(C)) \\
& =-\sum_{C \in \mathcal{D}} \rho\left(\lambda^{C} X(C)\right) \\
& \leq-\rho\left(\sum_{C \in \mathcal{D}}\left(\lambda^{C} X(C)\right)\right) \\
& \leq-\rho\left(\sum_{C \in \mathcal{D}}\left(\lambda^{C}\left(\left(u^{s}\left(q^{s}(C)\right)+\varepsilon\right)_{s \in S}\right)\right)\right) \\
& \leq-\rho\left(\left(u^{s}\left(\sum_{C \in \mathcal{D}} \lambda^{C} q^{s}(C)\right)_{s \in S}\right)+\sum_{C \in \mathcal{D}} \lambda^{C} \varepsilon 1^{S}\right) \\
& =-\rho\left(\left(u^{s}\left(\sum_{C \in \mathcal{D}} \lambda^{C} q^{s}(C)\right)_{s \in S}\right)\right)+\delta \varepsilon \sum_{C \in \mathcal{D}} \lambda^{C} \\
& \leq-\rho\left(X(D)+\delta \varepsilon \sum_{C \in \mathcal{D}} \lambda^{C}\right. \\
& =v^{D}(D)+\delta \varepsilon \sum_{C \in \mathcal{D}} \lambda^{C} .
\end{aligned}
$$

Since $\sum_{C \in \mathcal{D}} \lambda^{C} \leq n$, we have that $\delta \varepsilon \sum_{C \in \mathcal{D}} \lambda^{C}$ tends to zero as $\varepsilon$ tends to zero. Therefore, when taking the limit as $\varepsilon \downarrow 0$, the chain of equalities and inequalities (8)-(15) imply that

$$
\sum_{C \in \mathcal{D}} \lambda^{C} v^{D}(C) \leq v^{D}(D)
$$

so the game $\left(D, v^{D}\right)$ is balanced.

Equation (8) follows from Equation (5) and Equation (9) follows from the positive homogeneity of $\rho$. Inequality (10) is due to the subadditivity of $\rho$. Equation (11) plugs in Equation (6). Inequality (12) is true because of the positive homogeneity and superadditivity of $u$ (following from Proposition 2.9), and the monotonicity of $\rho$. Equation (13) makes use of the fact that $\rho$ satisfies translation invariance. Due to the monotonicity of $\rho$, Inequality (14) is true if we can prove that for each $s \in S$ it holds that

$$
u^{s}\left(\sum_{C \in \mathcal{D}} \lambda^{C} q^{s}(C)\right) \leq X^{s}(D) .
$$

Consider any $s \in S$. Take any $i \in D$, define $\mathcal{C}^{i}=\left\{C \in 2^{D} \mid i \in C\right\}$ and $\mathcal{D}^{i}=\mathcal{D} \backslash \mathcal{C}^{i}$. We have that

$$
\begin{aligned}
\sum_{C \in \mathcal{D}} \lambda^{C} u^{s}\left(q^{s}(C)\right) & =\sum_{C \in \mathcal{C}^{i}} \lambda^{S} u^{s}\left(q^{s}(C)\right)+\sum_{C \in \mathcal{D}^{i}} \lambda^{C} u^{s}\left(q^{s}(C)\right) \\
& \leq \sum_{C \in \mathcal{C}^{i}} \lambda^{C} u^{s}\left(q^{s}(C)\right)+\sum_{C \in \mathcal{D}^{i}} \lambda^{C} u^{s}(\theta(C)) \\
& \leq u^{s}\left(\sum_{C \in \mathcal{C}^{i}} \lambda^{C} q^{s}(C)+\sum_{C \in \mathcal{D}^{i}} \lambda^{C} \theta(C)\right) \\
& =u^{s}\left(\sum_{C \in \mathcal{C}^{i}} \lambda^{C}\left(t^{s}(C)+\theta(C)\right)+\sum_{C \in \mathcal{D}^{i}} \lambda^{C} \theta(C)\right) \\
& =u^{s}\left(\theta(D)+\sum_{C \in \mathcal{C}^{i}} \lambda^{C} t^{s}(C)\right) \\
& =u^{s}\left(\sum_{C \in \mathcal{C}^{i}} \lambda^{C}\left(\theta(D)+t^{s}(C)\right)\right),
\end{aligned}
$$


where the first inequality follows from Proposition 3.5 and the second inequality from Corollary 2.10.

We define $p^{s}(D)=\sum_{C \in \mathcal{C}^{i}} \lambda^{C}\left(\theta(D)+t^{s}(C)\right)$ and show that $p^{s}(D)+\theta(N \backslash D) \in L^{s}$. For every $C \in \mathcal{C}^{i}$ it holds that $q^{s}(C)+\theta(N \backslash C) \in L^{s}$. Therefore, it holds that

$$
\theta(D)+t^{s}(C)+\theta(N \backslash D)=q^{s}(C)+\theta(D \backslash C)+\theta(N \backslash D)=q^{s}(C)+\theta(N \backslash C) \in L^{s} .
$$

Since $\sum_{C \in \mathcal{C}^{i}} \lambda^{C}=1$ and $L^{s}$ is convex, we have that $p^{s}(D)+\theta(N \backslash D) \in L^{s}$.

Finally, we define $q^{s}(D) \in P$ by $q_{J}^{s}(D)=p_{J}^{s}(D)$ and

$$
q_{0}^{s}(D)=\ell^{s}\left(\theta_{0}(D), \sum_{C \in \mathcal{C}^{i}} \lambda^{C} t_{J}^{s}(C)\right) \text {. }
$$

Since $\ell^{s}$ is concave, we have

$$
q_{0}^{s}(D) \geq \sum_{C \in \mathcal{C}^{i}} \lambda^{C} \ell^{s}\left(\theta_{0}(D), t_{J}^{s}(C)\right)=\theta_{0}(D)-\sum_{C \in \mathcal{C}^{i}} \lambda^{C} t_{0}^{s}(C)=p_{0}^{s}(D),
$$

so $u^{s}\left(q^{s}(D)\right) \geq u^{s}\left(p^{s}(D)\right)$. By definition, it holds that $q^{s}(D) \in A^{s}(\theta(D))$. Assumption 2.7.(ii) implies that $q^{s}(D)+\theta(N \backslash D) \in L^{s}$. It follows that

$$
\sum_{C \in \mathcal{D}} \lambda^{C} u^{s}\left(q^{s}(C)\right) \leq u^{s}\left(p^{s}(D)\right) \leq u^{s}\left(q^{s}(D)\right) \leq X^{s}(D)
$$

Note that all the axioms of coherent measures of risk were used in the proof of Proposition 4.3 .

Not only is it true that all risk allocation games with liquidity constraints are totally balanced, but also any totally balanced game can be generated by a risk allocation game with liquidity constraints, by simply taking one perfectly liquid asset with a trivial liquidity policy and applying the construction of Csóka, Herings, and Kóczy (2009)[Proposition 3.2]. Thus we have the following theorem.

Theorem 4.4. The class of risk allocation games with liquidity constraints coincides with the class of totally balanced games, $\Gamma_{\mathrm{rl}}=\Gamma_{\mathrm{tb}}$.

\section{Conclusion}

Liquidity is of crucial importance when assessing the risk involved in an asset portfolio. Nevertheless, the literature on risk allocation games has so far ignored this important aspect. When we add a liquidity policy to a risk environment, we obtain an environment 
that is characterized by pervasive externalities. Indeed, when attributing the risk of a firm to its divisions, the question whether a single division satisfies the liquidity policy cannot be answered without making assumptions about the behavior of the complementary divisions.

We argue that the standard ways to deal with externalities, $\alpha$-effectiveness and $\beta$ effectiveness are not appropriate here, and that it is also not possible to simply ignore

the complementary divisions. Since in our framework the property rights of divisions are well-defined, it is meaningful to speak about inactivity of a coalition. We then say that a coalition of divisions satisfies the liquidity policy if it does so when the complementary coalition is inactive.

Our main result demonstrates that this approach leads to risk allocation games that are totally balanced. It is therefore possible to attribute the risk of a firm to its divisions in a stable way, since the core of a totally balanced game is evidently non-empty. In fact, the core of any subgame of a totally balanced game is non-empty as well, so the risk of any division can also be attributed in a stable way to its subdivisions too.

\section{References}

Acerbi, C., and G. Scandolo (2008), Liquidity risk theory and coherent measures of risk, Quantitative Finance 8, 681-692.

Artzner, P., F. Delbaen, J.-M. Eber, and D. Heath (1999), Coherent measures of risk, Mathematical Finance 9, 203-228.

Aumann, R.J. (1961), The core of a cooperative game without side payments, Transactions of the American Mathematical Society 98, 539-552.

Bondareva, O.N. (1963), Some applications of linear programming methods to the theory of cooperative games (in Russian), Problemy Kybernetiki 10, 119-139.

Buch, A., and G. Dorfleitner (2008), Coherent risk measures, coherent capital allocations and the gradient allocation principle, Insurance: Mathematics and Economics 42, 235 242.

Çetin, U., R.A. Jarrow, and P. Protter (2004), Liquidity risk and arbitrage pricing theory, Finance and Stochastics 8, 311-341.

Csóka, P., P.J.J. Herings, and L.Á. Kóczy (2007), Coherent measures of risk from a general equilibrium perspective, Journal of Banking and Finance 31, 2517-2534.

Csóka, P., P.J.J. Herings, and L.Á. Kóczy (2009), Stable allocations of risk, Games and Economic Behavior 67, 266-276. 
Denault, M. (2001), Coherent allocation of risk capital, Journal of Risk 4, 1-34.

Gillies, D.B. (1959), Solutions to general non-zero-sum games," in A.W. Tucker and R.D. Luce (eds.) Contributions to the Theory of Games IV, Annals of Mathematics Studies, 40, Princeton University Press, Princeton, pp. 47-85.

Homburg, C., and P. Scherpereel (2008), How should the joint capital be allocated for performance measurement?, European Journal of Operational Research 187, 208-227.

Jarrow, R.A., and P. Protter (2005), Liquidity risk and risk measure computation, Review of Futures Markets 11, 27-39.

Kalkbrener, M. (2005), An axiomatic approach to capital allocation, Mathematical Finance 15, 425-437.

Kim, J.H.T., and M.R. Hardy (2009), A capital allocation based on a solvency exchange option, Insurance Mathematics and Economics 44, 357-366.

Neumann, J. von, and O. Morgenstern (1944), Theory of Games and Economic Behavior, Princeton University Press, Princeton.

Predtetchinski, A., and P.J.J. Herings (2004), A necessary and sufficient condition for the non-emptiness of the core of a non-transferable utility game, Journal of Economic Theory $116,84-92$.

Schmeidler, D. (1969), The nucleolus of a characteristic function game, SIAM Journal on Applied Mathematics 17, 1163-1170.

Shapley, L.S. (1953), A value for n-person games, in H.W. Kuhn and A.W. Tucker (eds.), Contributions to the Theory of Games II, Annals of Mathematics Studies, 28, Princeton University Press, Princeton, pp. 307-317.

Shapley, L.S. (1967), On balanced sets and cores, Naval Research Logistics Quarterly 14, $453-460$. 